\title{
The encoding process of nonconfigural information in contextual cuing
}

\author{
Hirokazu Ogawa \\ University of Tokyo, Tokyo, Japan \\ AND \\ TAKatsune Kumada \\ National Institute of Advanced Industrial Science and Technology, Ibaraki, Japan
}

\begin{abstract}
Jiang and Wagner (2004) demonstrated that individual target-distractor associations were learned in contextual cuing. We examined whether individual associations can be learned in efficient visual searches that do not involve attentional deployment to individual search items. In Experiment 1, individual associations were not learned during the efficient search tasks. However, in Experiment 2, where additional exposure duration of the search display was provided by presenting placeholders marking future locations of the search items, individual associations were successfully learned in the efficient search tasks and transferred to inefficient search. Moreover, Experiment 3 demonstrated that a concurrent task requiring attention does not affect the learning of the local visual context. These results clearly showed that attentional deployment is not necessary for learning individual locations and clarified how the human visual system extracts and preserves regularity in complex visual environments for efficient visual information processing.
\end{abstract}

One of the most intriguing areas in the current visual attention literature concerns the relationship between attention and visual memory in information processing. Visual attention allows us to select a certain location or aspect of a scene and grant it priority in processing. Information extracted from the scene is stored in visual memory for further processing. Furthermore, recent studies have revealed that the visual system can utilize visual memory to make an efficient attentional selection of information. One type of memory-based attentional guidance is known as contextual cuing (Chun \& Jiang, 1998). In typical contextual-cuing experiments, participants have performed numerous inefficient visual search tasks that required a slow scrutiny of displays by serial attentional deployment. In half of the trials, the targets were embedded in configurations of distractors that were repeated throughout the experiment (repeated layouts); in the remaining trials, they were presented in novel configurations of distractors (nonrepeated layouts). In a given configuration, the target always appeared at the same location. The results have shown that the search reaction time (RT) for targets was shorter for the repeated layouts than for the nonrepeated ones, and the difference in RT intensified as the experiment progressed. This improved performance due to the repeated spatial layouts of displays is referred to as the contextual-cuing effect.

One important question that arises is what does the visual system extract and store as visual context through the repetitive presentation of the displays. It does not store target identity or its associated manual responses, because the target shape and orientation differ randomly on every trial. Furthermore, the contextual-cuing effect cannot be attributed to the learning of target locations per se, because contextual learning has occurred even when the target locations of the repeated layouts were identical to those of the nonrepeated ones. Thus, the visual system has to learn a consistent association between a repeated target location and repeated distractor locations in order to facilitate visual search.

Jiang and Wagner (2004) questioned whether the visual system extracts the pattern formed by all items (configural learning) or simply learns the location of each individual item (nonconfigural learning) as the visual context. They paired a given target location with two sets of distractor locations on separate trials and presented them repeatedly. After numerous practice trials, half the repeated layouts were made invariant, whereas the remaining half were reconstructed by recombining half of one trained distractor set with half of another trained distractor set. The results revealed that the contextual-cuing effect was transferred to the recombined layouts at a level that was the same as that for the normal repeated layouts, suggesting that individual locations of search items were learned as contextual information. Jiang and Wagner further examined whether learning the global configuration of distractors is necessary for the occurrence of contextual cuing by chang- 
ing individual item locations in the repeated layouts and preserving the learned global configuration. After being trained, the learned layouts were rescaled (expanded or contracted) or shifted (left, right, up, or down). Although the individual item locations no longer matched those seen during training, the participants searched faster with rescaled and shifted displays than with novel displays. This suggested that preserving a learned configuration permits the transfer of the contextual-cuing effect.

Another important question that arises is how does the visual system extract and learn invariant information in visual scenes. The inefficient search tasks used in the contextual-cuing literature appear to have involved attentional deployment toward individual items on display. Thus, it is plausible to assume that such an attentional shift may play a role in the nonconfigural learning of contextual cuing.

In support of this notion, Jiang and Song (2005) examined whether configural and nonconfigural contextual cuing could also occur in a change detection task. The results showed that although the repetition of item configuration improved change detection performance, the contextual-cuing effect that occurred during the change detection tasks failed to transfer to the recombined layouts. This suggested that nonconfigural learning did not occur in the change detection tasks. The results appear consistent with the notion that attentional deployment is necessary for nonconfigural learning, because one of the differences between a visual search task and a change detection task is the involvement of serial scanning of a display. Jiang and Song, however, pointed out many other important differences between them. For example, the visual search tasks in their experiments required attentional focus on a target location to discriminate its identity, whereas the change detection task emphasized the global features of the display and did not require focusing attention on local features. Thus, to clarify the mechanism of contextual learning in a visual search, the role of attentional deployment in the learning processes should be examined.

The aim of this article is to clarify whether nonconfigural learning of contextual cuing relies on serial deployments of visual attention. We examined whether individual associations can be learned during efficient visual searches that do not involve attentional deployment to individual search items. In Experiment 1, participants engaged in a learning phase wherein they performed efficient searches. This was followed by a test phase wherein they performed inefficient searches. A given target location was paired with two sets of distractor locations on separate trials, and they were repeatedly presented in the learning phase (repeated layouts). In the test phase, half the repeated layouts were made invariant; the other half were reconstructed by recombining half of one trained distractor set with half of another trained distractor set (recombined layouts). If nonconfigural learning of a visual context does not require attentional deployments during a visual search, the contextual-cuing effect that occurred in the learning phase can be transferred to the recombined layouts in the test phase.

\section{EXPERIMENT 1}

The aim of Experiment 1 was to examine, using an efficient visual search task, whether serial attentional deployment during visual searches is necessary for the occurrence of contextual cuing. The task required participants to search for a singleton target among distractors and discriminate the form of the target as quickly and accurately as possible. It allowed them to find the target without the serial scrutiny of visual attention.

\section{Method}

Participants. Thirty-two paid volunteers ( 13 of them male and 19 female; 19-27 years of age) having normal or corrected-to-normal visual acuity and normal color vision participated in the experiment after providing informed consent. None of them were aware of the purpose of this study.

Apparatus and Stimuli. All the stimuli were presented on a Sony Trinitron monitor ( $80-\mathrm{Hz}$ refresh rate). The experimental program was written using MATLAB with Psychophysics Toolbox extensions (Brainard, 1997; Pelli, 1997). A standard keyboard was used as a response device. The viewing distance was approximately $60 \mathrm{~cm}$. The display elements consisted of 11 outlined squares $\left(1.0^{\circ}\right.$ $\times 1.0^{\circ}$ ), with each square having a gap $\left(0.12^{\circ}\right)$ on one side (see Figure 1). The target square had a gap on its left or right side, with the distractor having a gap on either the top or the bottom. The displays comprised one target and 10 distractors. All the search items were randomly placed in the cells of a $10 \times 10$ virtual matrix subtending $12.5^{\circ} \times 12.5^{\circ}$ of visual angle.

Design and Procedure. An experimental session comprised two phases: a learning phase $(24$ trials $\times 15$ blocks $)$, wherein participants performed efficient search tasks, and a test phase (24 trials), wherein they performed inefficient search tasks. Each participant completed four experimental sessions successively. At the beginning of the learning phase of each session, 4 target locations were randomly selected from the matrix. These target locations shared the following three conditions: repeated, recombined, and nonrepeated. The target location of each condition was paired with two different sets of 10 distractor locations that were randomly selected from the matrix, resulting in eight layouts for each condition. In the nonrepeated layouts, the distractor locations were randomized for each presentation. The repeated layouts were invariant throughout the learning and test phases. The recombined layouts were repeated during the learning phase; however, after the learning blocks had been completed, they were reconstructed by swapping random halves of two trained distractor sets that had been paired with the same target location. Thus, the individual target-distractor pair from the learning phase was preserved, but the overall configuration was disrupted in the recombined layout. Since each experimental block comprised 24 trials ( 8 each for the repeated, recombined, and nonrepeated layouts) and each layout appeared once per block, each repeated and recombined layout was presented 15 times in a learning phase.

Each trial began with a fixation display presented for $750 \mathrm{msec}$, followed by the search display. The participants had to search for a target among the distractors and indicate the location of the gap in the target as quickly and accurately as possible. They were instructed to press the "c" or " $m$ " keys if the target had a gap on the left or the right side, respectively. A target was presented on each trial, and the identities of the targets and distractors were randomly selected for each trial. In the learning phase, the targets and distractors appeared in red and white, respectively, allowing the participants to search for the target without deploying attention to the distractor locations (efficient search). In contrast, all the search items in the test phase appeared in white, forcing attention to each item and making the participants perform inefficient searches (e.g., Han \& Kim, 2004). Following their responses or after the 4,000-msec response period had elapsed, the screen was cleared, and a new trial was initiated 
Learning phase (15 blocks)

Efficient search

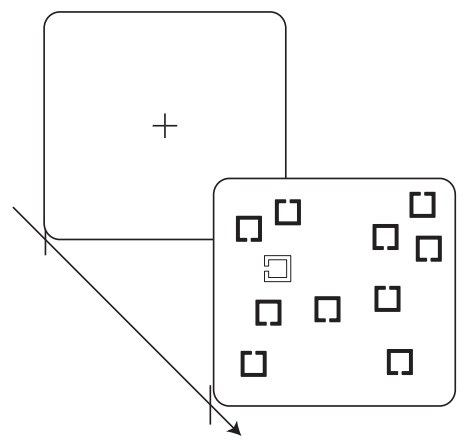

Test phase (1 block)

Inefficient search

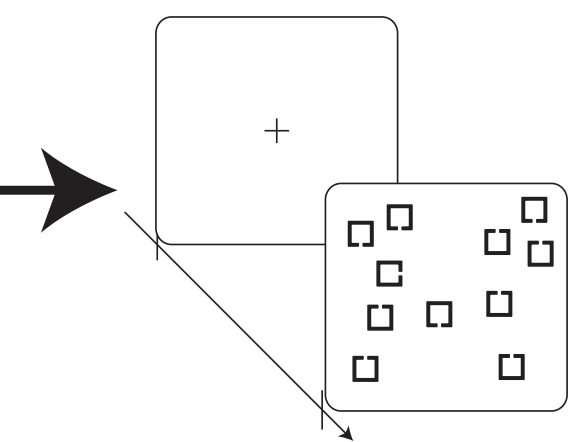

Figure 1. Sample displays from Experiment 1. In the learning phase (left panel), the targets and distractors were colored red and white, respectively, allowing the participants to search for the target without deploying attention to distractor locations (efficient search). However, in the test phase (right panel), all the search items were white, making the participants perform inefficient searches.

after a 750 -msec intertrial interval. Feedback was provided in the form of a prolonged low-pitched tone for errors.

\section{Results and Discussion}

The mean correct RT for each layout condition was calculated by collapsing the data across the four experimental sessions. Since the repeated and the recombined layouts were not distinguishable in the learning phases, these data were also collapsed for the analysis of the learning phases in the subsequent experiments. RTs shorter than $100 \mathrm{msec}$ were excluded from the analysis. Subjecting the remaining RTs to a recursive outlier elimination procedure with a moving criterion (Van Selst \& Jolicœur, 1994) resulted in the elimination of $1.9 \%$ and $0.4 \%$ of the learning and test phase trials, respectively. This procedure entails the removal of any RTs beyond $s$ standard deviations from the cell mean, with $s$ depending on the number of data points in the cell. Since the overall error rate was very low in both the learning phase (repeated layouts $=2.0 \%$, random layouts $=1.8 \%)$ and the test phase (repeated layouts $=$ $2.0 \%$, recombined layouts $=1.4 \%$, and random layouts $=$ $1.6 \%$ ), they were not analyzed further.

Figure 2 (left) shows the mean search RTs for each layout condition as a function of the number of repetitions (block). A two-way ANOVA was conducted with two within-subjects factors (layout, repeated or nonrepeated; repetition, $1-15)$. The analysis revealed a significant main effect of repetition $[F(14,434)=4.41, p<.001]$, reflecting the fact that overall RTs became slightly longer as the sessions progressed. No difference in RTs was found between the layout conditions $[F(1,31)=0.31, p>$ $.58]$, probably because of a ceiling effect. Otherwise, the
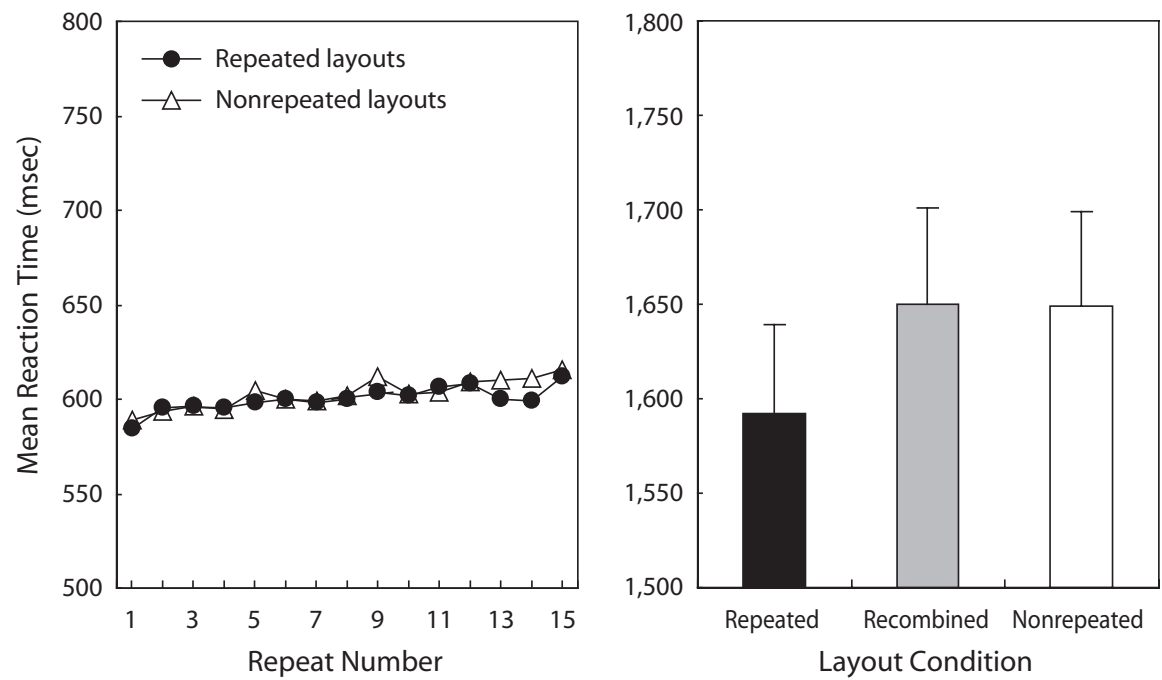

Figure 2. Results of Experiment 1. The left panel shows the learning phase, and the right panel shows the test phase. The error bars represent the standard errors of the means. 
number of repetitions might not be sufficient to exert the contextual-cuing effect in efficient search. Although the cause for the overall increase in RTs across blocks is unclear, we suspect that it was probably due to miscellaneous factors, such as boredom, fatigue, and frustration.

Figure 2 (right) shows the RTs in the test phase. As can be observed, the RTs for the repeated layouts were shorter than those in the other two conditions. The observation was statistically confirmed by a one-way ANOVA, which revealed a significant main effect of layout $[F(2,62)=7.59$, $p<.001]$. Post hoc tests (Tukey's HSD test) showed that the participants responded more quickly in the repeated layouts condition than in both the recombined and the nonrepeated layout conditions; no difference in RTs was observed between the latter two conditions (all $p \mathrm{~s}>.05$ ).

These data from the test phase clearly indicate that contextual cuing exerted its effect by training the participants to conduct efficient visual searches of repeated displays. To the best of our knowledge, this is the first research showing that, after training in an efficient search, context can be learned and the learning can be transferred to an inefficient search task. This finding provides a new perspective for the understanding of the implicit learning process in visual search because, so far, it has been assumed that deployment of attention is mandatory for the learning of contextual cuing. At the same time, we did not find any contextual advantage for the recombined layouts, suggesting that nonconfigural contextual cuing did not occur during efficient searches when the displays were repeated. Thus, these results can be interpreted to indicate that the learning of configural contextual cuing does not require attentional deployments; nevertheless, for the learning of nonconfigural contextual cuing, it is necessary to direct attention to individual locations of search items.

However, there remains another possibility to be considered. In this experiment, the presentation duration for the search displays was somewhat shorter than that in typical contextual-cuing experiments, because we used efficient searches wherein the participants could find the targets more easily. Thus, sufficient time to encode the individual locations of the distractors may not have been provided, which resulted in the failure to observe the nonconfigural contextual-cuing effect.

\section{EXPERIMENT 2}

Nonconfigural contextual cuing for recombined layouts did not occur in Experiment 1. There are at least two possible accounts for the finding. One is a search type account. That is, serial deployments of attention in a search display may be necessary for local learning of contextual cuing, so that nonconfigural learning can occur only in an inefficient search, not in an efficient search task. Alternatively, according to a duration account, the occurrence of local learning may depend on the time that an observer can use for encoding of a display, and not on the manner in which they direct their attention in a display. As was mentioned above, the RTs for the efficient searches in the training phase were quite a bit shorter than those for inefficient searches in which a nonconfigural contextual-cuing effect was observed (Jiang \& Wagner, 2004). Experiment 2 was designed to test these two possibilities.

In Experiment 2, placeholders predicting the future locations of search items were displayed before presentation of the search display in the training phases. These placeholders would allow the processes of contextual cuing to be initiated before the search display presentation and would provide additional time for the encoding of the individual locations of items. With this modification, two alternative accounts would predict different observations. According to the search type account, we should observe no local learning as long as search is parallel, no matter how long the display is presented, whereas the duration account would predict local learning in an efficient search when the duration of the display was extended.

\section{Method}

Participants. Twenty-four paid volunteers ( 20 of them male and 4 female; 19-27 years of age) participated in the experiment.

Apparatus and Stimuli. The apparatus and stimuli were identical to those in Experiment 1 .

Design and Procedure. All aspects of the design and procedure were identical to those Experiment 1, except that in the learning phase, the search display was preceded by the placeholder display (Figure 3). The placeholders appeared as white squares without a gap and marked the future locations of search items. The placeholder display was presented for $750 \mathrm{msec}$, followed by the search display, with no interstimulus interval. This exposure duration of the placeholders was determined from the difference in RTs between efficient and inefficient search tasks in a pilot study. The test phase proceeded exactly as in Experiment 1.

\section{Results and Discussion}

The mean correct RT was calculated the same way as in Experiment 1, with RTs shorter than $100 \mathrm{msec}$ being excluded from the analysis. Subjecting the remaining RTs to a recursive outlier elimination procedure with a moving criterion (Van Selst \& Jolicœur, 1994) resulted in the elimination of $1.9 \%$ and $0.8 \%$ of the learning and test phase trials, respectively. The overall error rate was very low in both the learning phase (repeated layouts $=1.6 \%$, random layouts $=0.9 \%$ ) and the test phase (repeated layouts $=0.9 \%$, recombined layouts $=1.3 \%$, and random layouts $=1.1 \%$ ).

Figure 4 (left) shows the mean search RTs for each layout condition as a function of the number of repetitions. An ANOVA was conducted with two within-subjects factors (layout, repeated or nonrepeated; repetition, 1-15). The analysis revealed a significant main effect of repetition $[F(14,322)=3.21, p<.001]$, reflecting the fact that the overall RTs became longer as the sessions progressed.

Figure 4 (right) shows the RTs in the test phase. An ANOVA on the transfer condition (repeated, recombined, or nonrepeated) revealed a significant main effect $[F(2,46)=5.44, p<.001]$. Post hoc tests (Tukey's HSD, all $p$ s $<.05$ ) showed that the RTs were significantly shorter for the repeated layouts than for the nonrepeated ones, suggesting that the contextual cuing in the efficient search tasks could be transferred to the inefficient search tasks. The RTs for the recombined layouts were shorter 


\section{Learning phase (15 blocks)}

Efficient search with placeholders

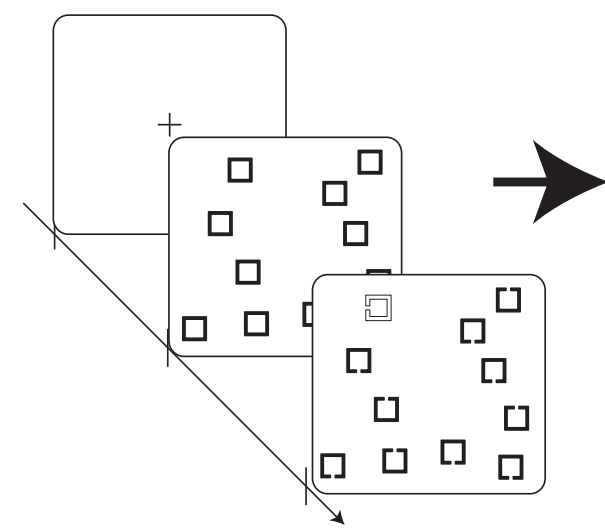

Test phase ( 1 block)

Inefficient search

Figure 3. Sample displays from Experiment 2. In the learning phase, the search display was preceded by a placeholder display. The placeholders appeared as white squares without a gap and marked the future locations of the search items. The placeholder display was presented for $750 \mathrm{msec}$, followed by the search display, with no interstimulus interval. The test phase proceeded exactly as in Experiment 1.

than those for the nonrepeated ones, suggesting that nonconfigural learning of contextual cuing occurred in the efficient search tasks. Finally, consistent with previous studies, the repeated and the recombined conditions did not differ significantly from each other (Jiang \& Song, 2005; Jiang \& Wagner, 2004). Although it is unclear why the preservation of nonconfigural information alone was sufficient for the preservation of configural information, this is suspected to have been due to a ceiling effect.

Experiment 2 differed from Experiment 1 in that search displays in the former were preceded by placeholders that were presented for $750 \mathrm{msec}$ in the learning phase. However, the results for the test phases were rather dif- ferent. Experiment 2 replicated the finding that configural contextual cuing occurred even when the participants performed efficient searches in the learning phase. More important, the result showed that RTs for recombined displays were shorter than those for nonrepeated displays. This indicates that nonconfigural contextual information could be learned through efficient searches performed in the learning phase when the exposure duration of the display was extended. These data clearly supported the duration account, not the search type account. That is, nonconfigural contextual cuing was unobserved in Experiment 1 , not because the learning of nonconfigural information required attentional deployment to the individual
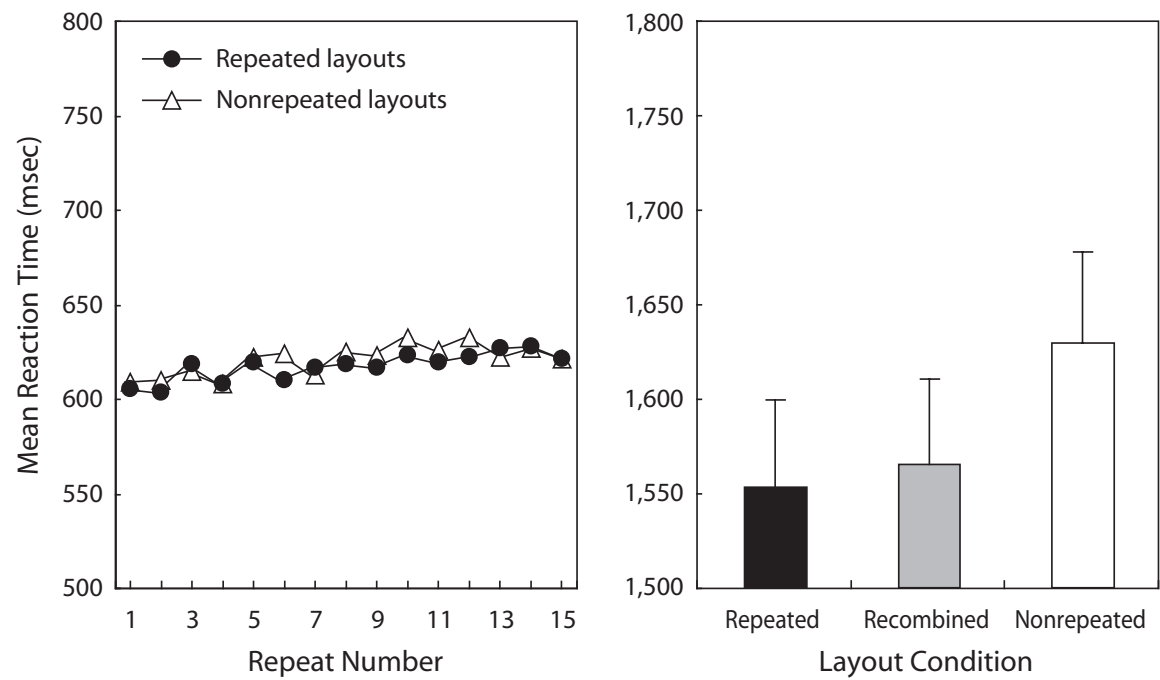

Figure 4. Results of Experiment 2. The left panel shows the learning phase, and the right panel shows the test phase. The error bars represent the standard errors of the means. 
locations included in an inefficient search, but because the display duration of the efficient search tasks conducted was insufficient to encode such information.

However, one potential concern with this experiment is that the participants may have focused on the locations of the placeholders while waiting for a search display, even if such a preview did not benefit target localization. Therefore, we designed Experiment 3, wherein the participants were asked to perform a central task that engaged their attention at a fixation point during a placeholder display.

\section{EXPERIMENT 3}

\section{Method}

Participants. Thirty-two paid volunteers (19 of them male and 13 female; 19-23 years of age) participated in the experiment.

Apparatus and Stimuli. The apparatus and stimuli were identical to those in Experiments 1 and 2 .

Design and Procedure. The design and procedure were the same as those in Experiment 2, except for the fact that an attention-demanding central task was added during presentation of the placeholder display in the learning phase (Figure 5). In Experiment 3, the fixation point flashed briefly (for $12.5 \mathrm{msec}$ ) three or four times during the placeholder display presentation. The participants had to count the number of flashes. Since this task was rather difficult and required them to focus on the fixation point, it was assumed that they did not focus on the search items during presentation of the placeholder display. This central task has also been used in previous works to prevent attentional deployment (e.g., Watanabe, 2005). After the participants had responded to the search target, the search display was erased, and the message " 3 or 4 ?" appeared at the center of the display. Without imposing a time limit, they were asked to press a key to indicate the number of times the fixation point had flashed. They pressed the "c" or " $\mathrm{m}$ " key if the fixation point flashed three and four times, respectively. The test phases proceeded exactly as in Experiments 1 and 2.

\section{Results and Discussion}

The mean correct RT was calculated the same way as those in Experiments 1 and 2, with RTs shorter than $100 \mathrm{msec}$ being excluded from the analysis. Subjecting the remaining RTs to a recursive outlier elimination procedure with a moving criterion (Van Selst \& Jolicœur, 1994) resulted in the elimination of $1.8 \%$ and $0.8 \%$ of the learning and test phase trials, respectively. The overall error rate was very low in both the learning phase (repeated layouts $=1.9 \%$, random layouts $=1.8 \%$ ) and the test phase (repeated layouts $=3.6 \%$, recombined layouts $=2.7 \%$, and random layouts $=2.4 \%$ ).

Figure 6 (left) shows the mean search RTs for each layout condition as a function of the number of repetitions. An ANOVA was conducted with two within-subjects factors (layout, repeated or nonrepeated; repetition, 1-15). The analysis did not reveal any significant main effects or interactions. Accuracy achieved for the central task was 79.7\% (repeated layouts) and 79.1\% (random layouts). These percentages were significantly above chance level and did not differ from each other.

Figure 6 (right) shows the mean RTs during the test phase. A one-way ANOVA revealed a significant main effect of layout $[F(2,62)=4.38, p<.05]$. Post hoc tests showed that RTs were significantly longer for the nonrepeated layouts than for the repeated ones, reflecting a contextual-cuing effect. They were also significantly longer for the nonrepeated layouts than for the recombined ones, suggesting that the nonconfigural association between targets and distractors was learned in the efficient search task even when a central task prevented focal attention from scanning a search array during presentation of a placeholder display. Finally, RTs
Learning phase (15 blocks)

Efficient search with a central task

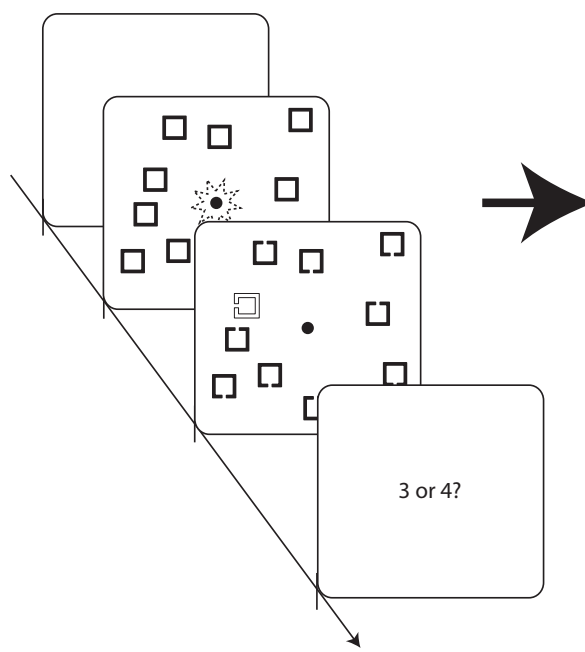

Test phase (1 block)

Inefficient search

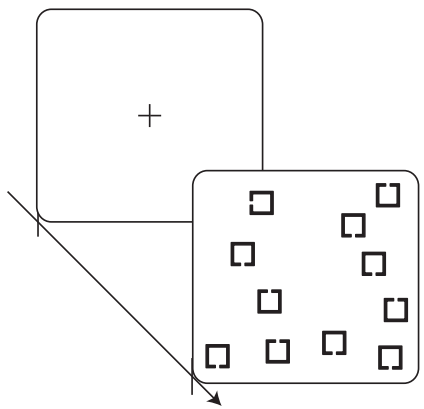

Figure 5. Sample displays from Experiment 3. In the learning phase, the fixation point briefly flashed three or four times during the presentation of the placeholder display. The participants were instructed to count the number of flashes. Since this task was rather difficult and required them to focus on the fixation point, it was assumed that they did not focus on the search items during presentation of the placeholder display. 

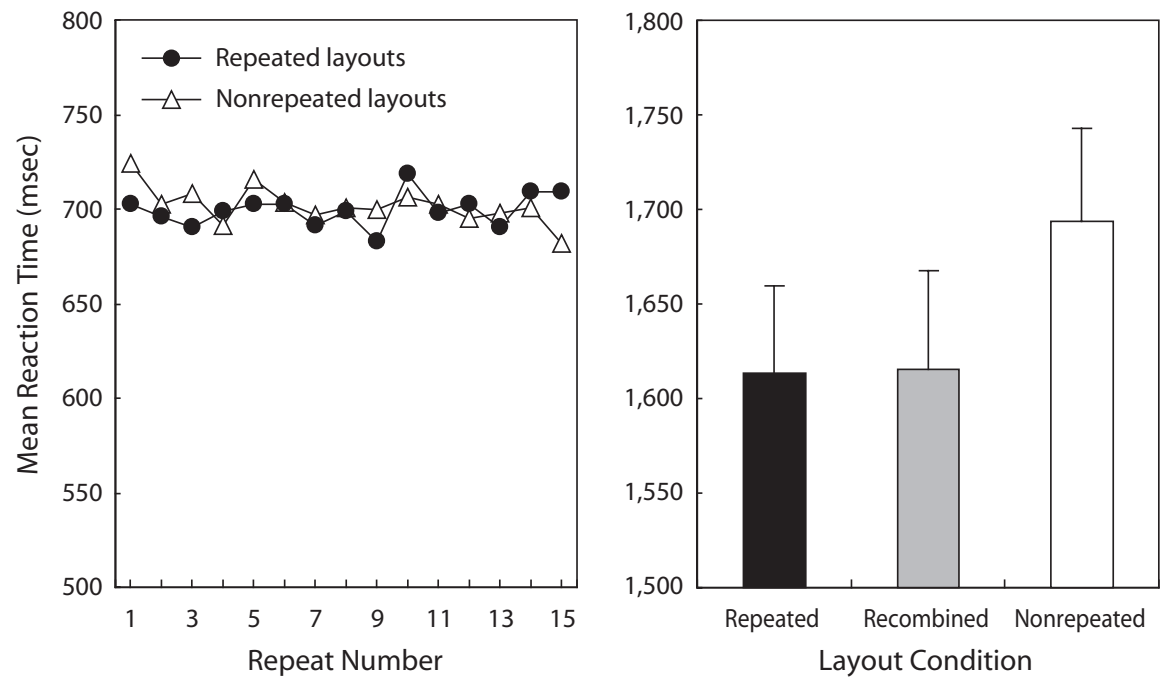

Figure 6. Results of Experiment 3. The left panel shows the learning phase, and the right panel shows the test phase. The error bars represent the standard errors of the means.

were not significantly different between the repeated and the recombined layouts (all $p$ s $>.05$ ).

Experiment 3 demonstrated that nonconfigural learning could occur even when participants focused on the central task during placeholder presentation in the learning sessions. This is inconsistent with the hypothesis that individual associations between target and distractor locations can be learned in efficient search tasks with placeholder display because attention is directed to the locations of the placeholders.

\section{GENERAL DISCUSSION}

A previous study had demonstrated that individual target-distractor associations are learned in contextual cuing (Jiang \& Wagner, 2004). In the present study, we examined whether individual associations can be learned in efficient visual searches that do not involve attentional deployment to individual search items. In Experiment 1, the participants engaged in a learning phase wherein they performed efficient searches. This was followed by a test phase wherein they performed inefficient searches. The results showed that learning was not transferred to a recombined layout, suggesting that they failed to learn the nonconfigural association between individual distractor locations and the target in the parallel search tasks. In Experiment 2, we tested the hypothesis that the lack of transfer in Experiment 1 occurred because the exposure duration for the displays, which was caused by shorter RTs for the parallel search tasks, was too short for nonconfigural learning involving the encoding of individual locations in the search displays. When a placeholder's display preceded presentation of a parallel search display, which allowed sufficient time to encode individual associations, we found the nonconfigural contextual-cuing effect in the test phase, which supported the hypothesis. Furthermore, Experiment 3 showed that the lack of transfer to the re- combined condition should not be attributed to attentional deployments to the locations of the search items during presentation of a placeholder display. Together, these results provide evidence that the learning association between individual distractor and target locations depends on the exposure duration of a search display, not on attentional deployments to search items.

This study revealed that a long temporal window was required also for nonconfigural learning in a contextualcuing task; however, it is important to determine why this is so. A process requiring a long temporal lag should not be associated with sensory memory. We speculate that the contextual information for displays may be encoded in a global-to-local manner. That is, under a shorter presentation duration for a display, global information (i.e., configural information) for the display may be dominantly encoded. In contrast, under a longer presentation duration for a display, contextual information may be more deeply processed, allowing the encoding of local information (i.e., individual associations). Thus, our results are consistent with the notion that global-to-local precedence in visual processing is implicated in certain visual tasks (Navon, 1977) and coarse-to-fine processing is implicated in natural scene recognition (Oliva \& Schyns, 1997; Schyns \& Oliva, 1994). In the present experiments, the nonconfigural cuing effect appears to have occurred in an all-or-none fashion: No cuing effect was found for the exposure duration of $600 \mathrm{msec}$ (Experiment 1), whereas an extended presentation duration of $750 \mathrm{msec}$ provided a complete cuing effect (Experiments 2 and 3 ). This implies that information is not transferred into long-term memory only so long as consolidation in visual short-term memory proceeds to some extent and that such a critical time point may exist somewhere between 600 and $1,550 \mathrm{msec}$ from onset of the display. How long a presentation duration would be enough to allow learning of the nonconfigural context? If presentation duration for the display is the crit- 
ical factor for local learning, would a limited search duration reduce local learning even in an inefficient search? These questions need to be answered in future research.

Although such mechanisms may also occur in contextual cuing, we do not insist that such a global-to-local processing is mandatory for contextual cuing, because it depends on various factors, such as the physical properties of the stimulus or the diagnosticity of a particular spatial scale that is sufficient for a given task. Our tasks do not necessarily appear to be dependent on the local features of the display; furthermore, such an information demand might bias the encoding process toward operating at the most informative scale available for the task. Jiang and Song (2005) have consistently reported that training in a change detection task requires attentional resources for the global features of a display, rather than for the local features produced only by configural learning, but does not require resources for the features produced by nonconfigural learning. A task demand or attentional set in a given task could modulate resource allocation between different types of context learning. The relationship between task demand and the learning process for contextual cuing is still unclear and, therefore, is an important goal for future research.

To summarize, this study provides the first evidence that an efficient search is sufficient for the learning of contextual cuing. We have clarified one factor that determines whether learning of the individual locations of search items can occur. When training involves efficient search with shorter display presentation durations, the visual system can learn the global configuration of the search items. However, when training involves efficient search with a sufficient exposure duration, both global layout and individual associations between target and distractor locations are extracted and retained. We also demonstrated that a concurrent task requiring focus of attention does not affect the learning of local visual context. These results clearly show that attentional deployment is not necessary for the learning of the individual locations of search items and shed light on how the human visual system extracts and preserves regularity in complex visual environments for efficient visual information processing.

\section{AUTHOR NOTE}

This research was supported by the Research Fellowships of the Japan Society for the Promotion of Science for Young Scientists, granted to H.O. We thank Tom Carr, Yuhong Jiang, and Melina Kunar for helpful comments. Correspondence concerning this article should be addressed to H. Ogawa, Cognitive Science Research Group, Research Center for Advanced Science and Technology, University of Tokyo, 4-6-1 Komaba, Meguro-ku, Tokyo 153-8904, Japan (e-mail: ogawa@fennel.rcast.u -tokyo.ac.jp).

\section{REFERENCES}

Brainard, D. H. (1997). The Psychophysics Toolbox. Spatial Vision, 10, 433-436.

Chun, M. M., \& Jiang, Y. (1998). Contextual cueing: Implicit learning and memory of visual context guides spatial attention. Cognitive Psychology, 36, 28-71.

Han, S. H., \& Kim, M. S. (2004). Visual search does not remain efficient when executive working memory is working. Psychological Science, 15, 623-628.

JIANG, Y., \& SoNG, J.-H. (2005). Spatial context learning in visual search and change detection. Perception \& Psychophysics, 67, 1128-1139.

JiAnG, Y., \& WAGNER, L. C. (2004). What is learned in spatial contextual cuing - configuration or individual locations? Perception \& Psychophysics, 66, 454-463.

NAvon, D. (1977). Forest before trees: The precedence of global features in visual perception. Cognitive Psychology, 9, 353-383.

Oliva, A., \& Schyns, P. G. (1997). Coarse blobs or fine edges? Evidence that information diagnosticity changes the perception of complex visual stimuli. Cognitive Psychology, 34, 72-107.

Pelli, D. G. (1997). The VideoToolbox software for visual psychophysics: Transforming numbers into movies. Spatial Vision, 10, 437-442.

Schyns, P. G., \& Oliva, A. (1994). From blobs to boundary edges: Evidence for time- and spatial-scale-dependent scene recognition. Psychological Science, 5, 195-200.

VAN Selst, M., \& Jolicceur, P. (1994). A solution to the effect of sample size on outlier elimination. Quarterly Journal of Experimental Psychology, 47A, 631-650.

Watanabe, K. (2005). Asymmetric mislocalization of a visual flash ahead of and behind a moving object. Perception, 34, 687-698.

(Manuscript received June 29, 2006; revision accepted for publication September 10, 2007.) 